\title{
Adherence intervention for HIV-infected persons who use drugs: adaptation, open trial, and pilot randomized hybrid type 1 trial protocol
}

\author{
Kasey Claborn ${ }^{1,2^{*}}$, Sara Becker ${ }^{3,4}$, Don Operario ${ }^{4}$, Steve Safren ${ }^{5}$, Josiah D. Rich ${ }^{2,6}$ and Susan Ramsey ${ }^{2,3}$
}

\begin{abstract}
Background: HIV-infected people who use drugs (PWUD) exhibit the highest rates of non-adherence to antiretroviral therapy (ART) among people living with HIV. This contributes to poor treatment outcomes, increased morbidity and mortality, and HIV transmission. However, current interventions fail to address the unique barriers to adherence faced by this population. Life Steps is a brief, single session intervention that demonstrated increased ART adherence among HIV-infected individuals. This study protocol seeks to improve clinical practice by adapting Life Steps for HIVinfected PWUD and adding a brief motivational intervention addressing drug use. This intervention will incorporate educational, motivational, and behavioral skills components specifically aimed at improving adherence and linkage to substance use treatment among HIV-infected PWUD.
\end{abstract}

Methods: This project will consist of three phases using a mixed-methods approach. In Phase 1, qualitative interviews with HIV-infected PWUD and community providers, coupled with feedback from an expert review panel, will be used to enhance the existing Life Steps manual and interventionist training protocol. In Phase 2, the prototype will be pilot tested and qualitative exit interviews with HPWUD will identify the strengths and limitations of the intervention. Data regarding feasibility, acceptability, and barriers to delivery will guide modifications to finalize a modified Life StepsDrug Use (LS-DU) protocol. In Phase 3, a pilot type 1 hybrid effectiveness-implementation trial will examine the effectiveness of LS-DU relative to a health education intervention control condition on ART adherence and viral load data at 1-, 3-, and 6-months. Data regarding clinic readiness for implementation and intervention sustainability potential will be collected.

Discussion: This protocol will adapt and evaluate an intervention to improve adherence among HIV-infected PWUD. Results of this study will provide significant data on the acceptability, initial effectiveness, and sustainability potential of an adherence intervention for a high risk and underserved population.

Trial registration NCT02907697

Keywords: HIV/AIDS, Substance use, Adherence, Motivational interview, Mixed methods, Protocol

\section{Background}

Illicit drug use remains a common and significant problem among people living with HIV (PLWH). Drug use

\footnotetext{
*Correspondence: kasey.claborn@austin.utexas.edu

1 Department of Psychiatry, The University of Texas at Austin Dell Medical

School, 1912 Speedway Austin, TX, USA

Full list of author information is available at the end of the article
}

is one of the most significant barriers to antiretroviral therapy (ART) adherence, contributing to suboptimal treatment outcomes and increased transmission of HIV. As suggested by the syndemics model [1], multiple morbidities such as drug use and HIV act synergistically to produce poorer health outcomes. Over $81 \%$ of persons living with HIV report a history of illicit drug use and nearly one in four meet diagnostic criteria for a severe 
substance use disorder [2]. Drug use remains prevalent among PLWH enrolled in primary care with one study showing that $24 \%$ report marijuana use, $9 \%$ report amphetamine use, $8.5 \%$ report crack cocaine use, $10 \%$ report polydrug use, and almost $3 \%$ report injection drug use in the preceding 3 months [3]. This study was conducted using the CFAR Network of Integrated Clinical Systems (CNICS) data which consisted of academically affiliated HIV clinics; thus, it is possible that these are underestimates of active illicit drug use. These data demonstrate that sustainable interventions and risk reduction programs addressing substance use are needed within the HIV clinical setting.

People who use drugs (PWUD) experience unique challenges with HIV treatment and adherence. HIV-infected PWUD are more likely to experience delayed treatment, suboptimal service utilization, increased rates of HIV transmission in the community, and inferior treatment outcomes [4-6]. Relative to PLWH who do not use drugs, HIV-infected PWUD are more than twice as likely to be non-adherent to ART [7]. Both interpersonal and social factors limit adherence in this population: HIVinfected PWUD are less likely to have primary care providers or strong patient-provider relationships and have lower levels of social support $[7,8]$. Socioeconomic factors prevalent in PLWH, including lack of stable housing and medical insurance, poor education and low literacy, and low health literacy pose further barriers to adherence. HIV-infected PWUD are also more likely to have medical (e.g., hepatitis $C$, tuberculosis) and psychiatric (e.g., depression, anxiety) comorbidity, neurocognitive impairment, and increased risk for drug overdose, which contribute to reduced adherence and poorer health outcomes [8].

\section{Prior adherence interventions for HIV-infected people who use drugs}

Several intensive treatment approaches have been shown to improve adherence among HIV-infected PWUD including the integration of HIV care and intensive drug use treatment, directly administered ART doses at the clinic, contingency management, and peer-driven interventions [6]. Although these interventions are effective in the short-term, adherence improvements are frequently not maintained after the direct observation has been terminated [9] and such intensive interventions may not be feasible or sustainable in clinics with limited resources, which dampens the potential for widespread implementation [10]. Additionally, these interventions do not teach HIV-infected PWUD the behavioral skills needed to maintain adherence once it is achieved. Behavioral interventions overcome these limitations by teaching adherence and problem-solving skills, using brief motivational interviewing (BMI) and cognitive behavioral approaches to sustain behavior change. These interventions have been successfully augmented with booster sessions and delivered by trained clinic nurses and staff. This approach has shown promise in promoting ART adherence and self-efficacy to adhere among PLWH, but evidence is severely limited by use of small sample pilot and case studies. To date, only one behavioral adherence intervention-the Life Steps intervention-has been evaluated in HIV-infected PWUD.

Life Steps was developed by Safren and colleagues using motivational interviewing and cognitive behavioral techniques to teach the following: facts about ART and HIV, problem-solving around transportation to clinic appointments and obtaining medications, skills to improve communication with healthcare providers, strategies to cope with side effects, development of a daily medication schedule, storage of medications away from home, reminder cues for pill-taking, and responding to slips in adherence. This model consists of a brief, single session intervention that has been evaluated as both a stand-alone intervention and an adjunct to more intensive cognitive behavioral therapy (CBT). Data from multiple studies indicates that Life Steps and similar interventions are associated with increased adherence relative to self-monitoring in PLWH [7], though metaanalyses suggest that these effects are modest across studies $[11,12]$.

\section{Limitations of life steps with HIV-infected people who use drugs}

Prior research on the Life Steps protocol with HIVinfected PWUD is subject to several limitations. First, only one study to date has evaluated Life Steps among a drug using population and this study focused on depressed injection drug users who are unlikely to be representative of the full range of HIV-infected PWUD [13]. Additionally, both study conditions received Life Steps; the experimental condition also received CBT for Adherence and Depression which consisted of seven CBT-focused modules. Second, no prior studies have examined which specific components of the Information-Motivation-Behavioral Model are likely to be most relevant for HIV-infected PWUD and if additional skills modules related to illicit drug use should be incorporated. Third, the Life Steps protocol focuses on skills acquisition and behavior modification related to HIV medication taking behavior, but does not address substance use or linkage to addiction treatment. Brief motivational interventions (BMIs) are especially well suited to this population, as both adherence to HIV treatment and drug use have been related to patient motivation for change [14]. Finally, there has been virtually no research 
examining barriers and facilitators of the implementation of behavioral ART adherence interventions within real-world clinics. Such research is essential in order to accelerate the translation of research into practice and improve the outcomes of this hard to reach, high risk population.

\section{Protocol objective and specific aims}

The overarching objective of the current protocol is to adapt a behavioral adherence intervention for HIVinfected PWUD and obtain data on its preliminary effectiveness and implementation potential. The proposed project consists of three phases (see Table 1). In Phase 1, qualitative interviews with HIV-infected PWUD and community providers, coupled with feedback from an expert review panel, will be used to adapt the existing Life Steps manual and community clinician training protocol. In Phase 2, qualitative data from phase 1 will inform development of the intervention manual. The protocol will be pilot tested and in-depth qualitative interviews with HIV-infected PWUD and community clinicians will be used to make final refinements to the adapted Life Steps-Drug Use (LS-DU) model. In Phase 3, a pilot type 1 hybrid effectiveness-implementation trial will examine the effectiveness of LS-DU relative to a health education control condition on ART adherence and viral load data at 1-, 3-, and 6-months. Data regarding clinic readiness for implementation and intervention sustainability potential will be also be collected. Combined, these three phases will address three study aims:

Table 1 Clinical trial schedule of enrollment, interventions, and assessments

\begin{tabular}{|c|c|c|c|c|c|}
\hline \multirow[b]{3}{*}{ Timepoint** } & \multicolumn{5}{|c|}{ Study period } \\
\hline & \multirow{2}{*}{$\begin{array}{c}\text { Enrollment } \\
-t_{1}\end{array}$} & \multirow{2}{*}{$\begin{array}{c}\text { Allocation } \\
0\end{array}$} & \multicolumn{3}{|c|}{ Post-allocation } \\
\hline & & & $t_{1}$ & $t_{2}$ & $t_{3}$ \\
\hline \multicolumn{6}{|l|}{ Enrollment } \\
\hline Eligibility screen & $x$ & & & & \\
\hline Informed consent & $x$ & & & & \\
\hline Allocation & & $x$ & & & \\
\hline \multicolumn{6}{|l|}{ Interventions } \\
\hline Life steps-drug use & & & 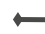 & & $\rightarrow$ \\
\hline Health education & & & $\hookleftarrow$ & & $\longrightarrow$ \\
\hline \multicolumn{6}{|l|}{ Acceptability } \\
\hline Acceptability & & & & & $x$ \\
\hline Effectiveness & $x$ & $x$ & $x$ & $x$ & $x$ \\
\hline Implementation & $x$ & & & & $\mathrm{x}$ \\
\hline
\end{tabular}

Aim 1: To adapt the Life Steps intervention by incorporating a BMI focused on linkage to substance use treatment, and tailoring the skills to meet the unique needs of HIV-infected PWUD. Aim 2: To standardize the adapted intervention into a manual and train community-based intervention facilitators. Aim 3: To obtain data on the preliminary effectiveness and implementation potential of the adapted intervention relative to a health education control.

\section{Methods \\ Participants}

A total of up to 84 patients and 35 clinicians will participate across three phases: 6 patients and 29 clinicians participated in Phase 1 (qualitative interviews), 18 patients and 6 clinicians will be recruited in Phase 2 (pilot testing), and 60 patients will be recruited in Phase 3 (pilot randomized hybrid trial). The primary objective for Phase 3 will be to determine a reasonable effect size for LS-DU rather than to determine statistical significance between groups. With 30 participants per group and effect size range of $\mathrm{d}=.40$ to .60 , power to detect the difference ranges from .54 to .75 . With $\mathrm{N}=60$ we will have .80 power to detect effect sizes in the medium-large range above $\mathrm{d}=.65$. Effect size estimates will include odds ratios for ART adherence and squared semi partial correlations for repeated continuous measures of putative mechanisms.

\section{Inclusion criteria}

Patients will be eligible for each phase of the study if they are (1) $\geq 18$ years of age, (2) HIV-infected as confirmed by medical record review, (3) prescribed an ART regimen, and (4) meet DSM-V criteria for a substance use disorder (other than tobacco, marijuana, and alcohol) over the past 90 days. Although heavy alcohol consumption affects ART adherence and cannabis use has demonstrated mixed results in the literature, we chose to narrow the target population to illicit drug use to have a more homogenous sample for this pilot trial. In Phase 3 (pilot randomized trial), participants must also report $<100 \%$ adherence to ART regimen and have experienced a detectable viral load $(>20$ copies $/ \mathrm{mL}$ ) within the last 6 months. Our eligibility criteria for a substance use disorder is broad given the LS-DU will focus on improving ART adherence and linking patients to appropriate substance use treatment resources. Our goal is to create an intervention that is easily implemented in clinical settings. We will examine qualitative data from Phase 1 to assess the potential need for the intervention to take a more narrow approach and adjust the manual development based on these data.

Clinicians were eligible to participate in Phase 1 provider interviews if they were: over the age of 18 , employed 
at a local HIV clinic or substance use treatment facility, and had $>6$ months experience working with patients living with HIV and/or patients with a substance use disorder.

\section{Exclusion criteria}

Exclusion criteria for all study phases include issues that jeopardize informed consent, including cognitive impairments, active psychosis, current suicidal ideation, and not being fluent in English. Patient participants will be administered a brief measure of capacity to consent [15].

\section{Study enrollment and randomization}

In accordance with institutional review board procedures, participants will be recruited from an academic affiliated HIV treatment center which is funded by the Ryan White program and situated in an urban region. We will hang flyers for each phase of the study to advertise study details. A research assistant will pre-screen medical records of patients with clinic appointments and identify those who appear to be eligible. Written informed consent will be obtained from those interested in participating.

\section{Phase 1 procedures: qualitative methods to inform intervention adaptation Overview}

Individual interviews have been conducted with 29 treatment providers ( $\mathrm{n}=16$ HIV providers; $\mathrm{n}=13$ substance use providers) and six PLWH who met eligibility criteria to gather feedback on the Life Steps protocol, ideas for adapting the protocol to PWUD, and training preferences. Interview topics included: (1) current support for HIV care within the clinic or community for HPWUD, (2) brainstorming intervention content, (3) critique of proposed intervention content and existing materials, and (4) evaluation of intervention length, intensity, frequency, and mode of delivery. Patient and provider data are being analyzed and triangulated to inform adaptation of the Life Steps intervention to HIV-infected PWUD. Patients and providers received $\$ 40$ for participating in a 60-90 min qualitative interview.

\section{Manual development}

The Life Steps manual will be enhanced to address the most salient needs of HIV-infected PWUD while retaining the core elements of the intervention. Data from Phase 1 will guide modification of the manual. The manual will be evaluated based on the theoretical model, targeted outcomes, therapeutic strategies, and content. Substantive Changes Expected. Based on extant literature, there are two key areas in which substantive changes are expected in the adaptation of the Life Steps manual (see
Table 2): Content. The Information-Motivation Behavioral Model [16] will guide content targeting individual knowledge, motivation, and skills development (see Fig. 1). Considering the synergistic interaction of HIV and drug use on nonadherence, syndemics theory [1] will guide the adaptation of intervention content to address contextual challenges of HIV-infected PWUD (e.g., stigma, stress, poverty). Delivery. Based on the hypothesized outcomes from Phase 1, we expect the adapted LS-DU protocol will contain a new drug use module focused on building motivation for change and linkage to substance use treatment; this module will be informed by best practices in the BMI literature. While we have anticipated preliminary adaptations based on the extant literature, changes to the protocol will be based on data obtained during the qualitative phase which will be sufficiently broad and open to assess for factors that we have not anticipated. Once the manual is developed, a review panel of five experts in HIV, addiction, and ART adherence will provide feedback about the intervention manual. We will use an affinity grouping procedure to merge similar recommendations. The draft of recommendations will be reviewed through two rounds of preliminary ratings, teleconference discussions, and written comments to result in a set of final recommendations which will inform revision and finalization of the manual.

\section{Phase 2 procedures: piloting and training of community-based facilitators Therapist training, fidelity, and competence}

Following manual refinement, two master's-level community clinicians will be trained in fidelity and competence with the intervention protocol. Training will consist of a half-day didactic workshop, followed by video-taped role plays, which will be coded by two independent raters for fidelity and competence. We will assess competence using the Motivational Interviewing Treatment Integrity 2.0 (MITI) [17] and the Yale Adherence and Competency Scale [18]. Following training, clinicians will provide feedback regarding their training experience and recommendations for the training protocol.

\section{Patient qualitative interviews}

The two trained clinicians will deliver the LS-DU intervention to 8-12 HIV-infected PWUD using an iterative process. We will conduct exit interviews with patients and the two clinicians following completion of the intervention to assess intervention acceptability and feasibility. Data collected during the individual interviews will include: (1) critique of intervention content and materials, (2) evaluation of intervention length, intensity, frequency, and mode of delivery, and (3) suggestions to further enhance the intervention. Patients will again 


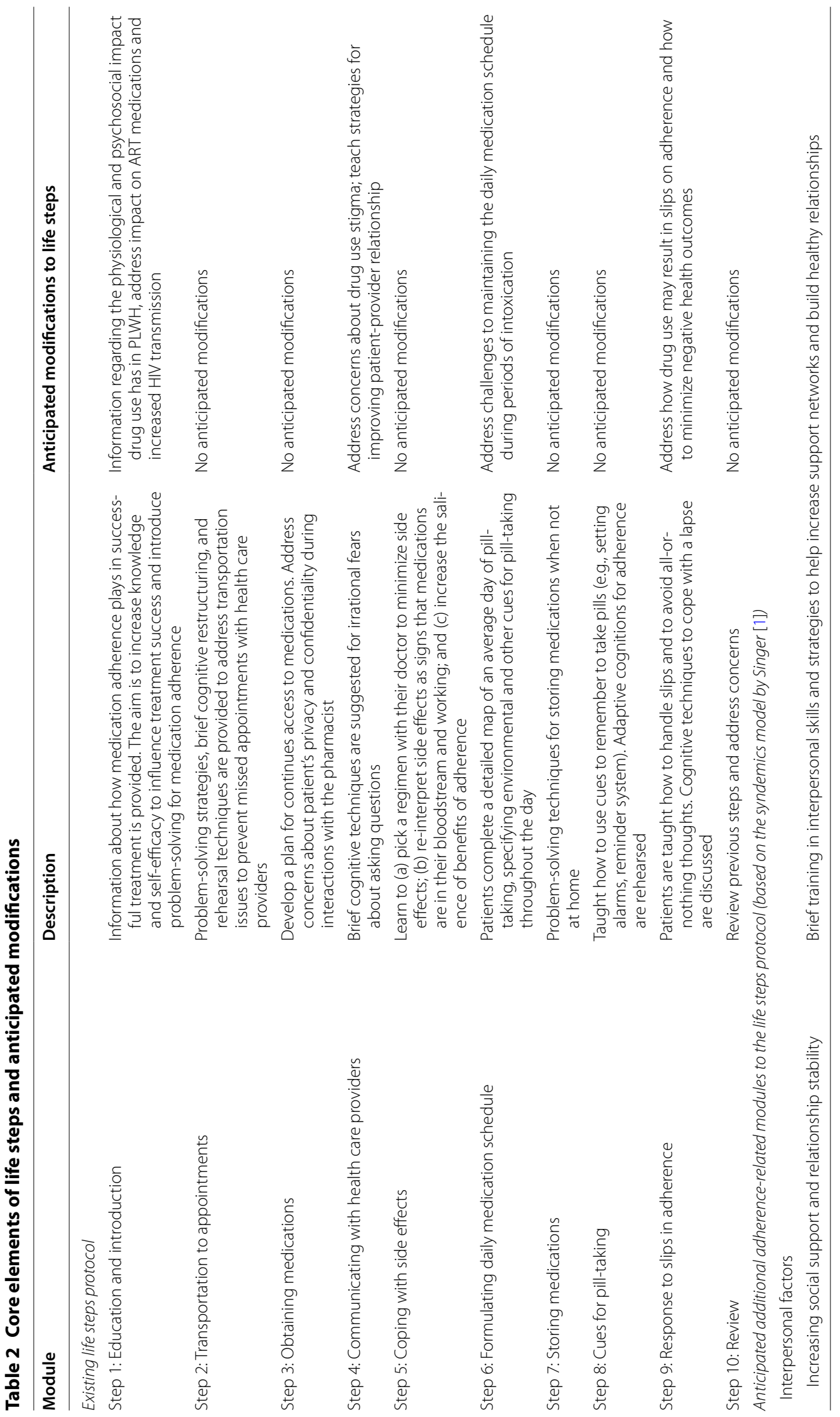




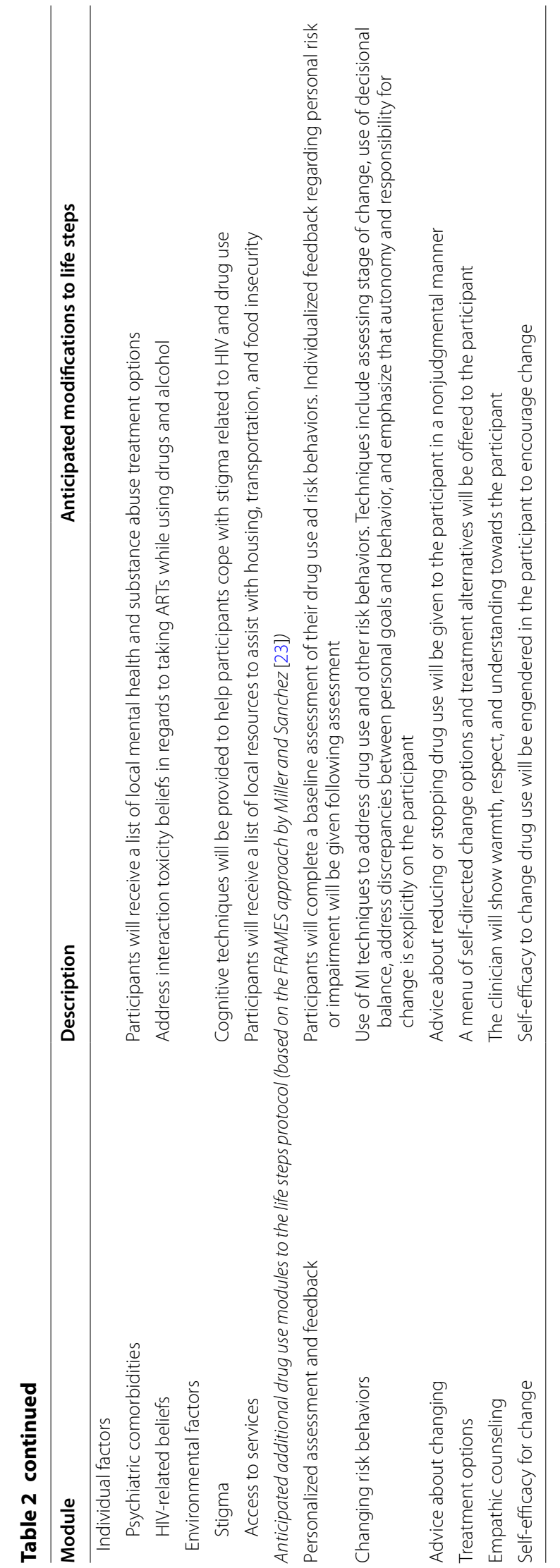




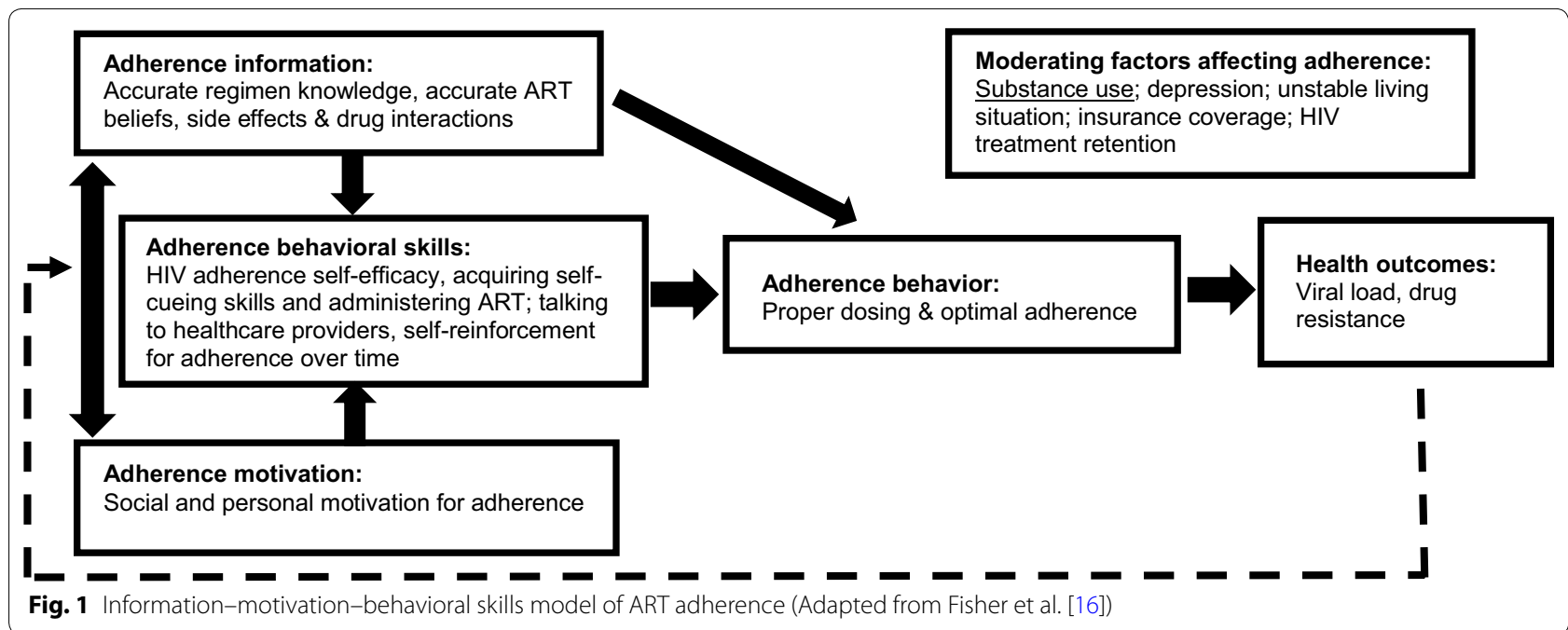

receive $\$ 40$ for participating in a 60 -min qualitative interview. Interviews will be administered until saturation is reached.

\section{Phase 3 procedures: type 1 hybrid effectiveness-implementation pilot trial Overview}

Following the open trial, a pilot type 1 hybrid randomized effectiveness-implementation trial will be conducted to determine the effectiveness of the LS-DU protocol relative to health education. Hybrid effectiveness-implementation trials are designs that simultaneously gather data on treatment effectiveness and implementation outcomes [19]. In a type 1 hybrid trial, the primary emphasis is on the evaluation of treatment effectiveness, and the secondary emphasis is on the collection of data on implementation outcomes. Because the current study is designed to collect pilot data for a future study, measures of acceptability and feasibility are defined as the primary outcomes. Measures of effectiveness are then defined as the secondary outcomes, while data on implementation effectiveness are gathered as an exploratory outcome.

All participants will complete a baseline assessment consisting of a series of self-report measures and a urine toxicology screen. Following completion of the baseline assessment, participants will begin 2 weeks of baseline ART adherence data collection using an electronic pill cap monitoring system (MEMScaps). Only after completing the MEMS data collection will patients be randomly assigned to treatment condition using urn randomization [20] controlling for gender and viral load.

\section{Treatment conditions}

Participants will be randomized to either the LS-DU experimental intervention or the health education control. We considered using the original Life Steps as a comparator condition; however, we believe that a minimally active, but ethical control group is the most direct test of the intervention (relative to treatment as usual). A more active control intervention, such as the original Life Steps protocol, may attenuate potential effects and would require a much larger sample size. The LS-DU intervention will utilize the manual refined at the end of Phase 2. Health education is a time-matched control condition that covers a variety of general health topics and has proven to be a credible control condition in prior studies [21]. Intervention clinicians will be given a health education training manual and will complete videotaped role plays. Two independent coders will assess fidelity to the health education intervention using a topic checklist. Both conditions will consist of two in-person intervention sessions of 60 min duration separated by 1 week. The LS-DU intervention will also consist of two follow-up booster phone sessions at 1 and 2 months following the last in-person session.

\section{Assessments}

With the exception of demographic data, all of the measures described below will be administered at baseline and the three follow-up assessments. To enhance retention, participants will receive an escalating compensation schedule in gift cards for completion of the 1-, 3-, and 6-month follow-ups. Participants will receive a breathalyzer test prior to each assessment, and impaired participants will be rescheduled. 
Table 3 Outcome variables and assessment points

\begin{tabular}{|c|c|c|c|c|}
\hline Quantitative measures & Stakeholder & BL & 1-, 3-mos follow-up & 6-mos follow-up \\
\hline Demographics & All & $x$ & & \\
\hline \multicolumn{5}{|l|}{ Acceptability } \\
\hline Program satisfaction questionnaire & Patients, clinicians & & & $x$ \\
\hline \multicolumn{5}{|l|}{ Effectiveness } \\
\hline Medication adherence (MEMS) & Patients & $x$ & $x$ & $x$ \\
\hline Viral load & Patients & $x$ & & $X$ \\
\hline Substance use & Patients & $x$ & $x$ & $x$ \\
\hline \multicolumn{5}{|l|}{ Implementation } \\
\hline Organizational readiness for change assessment tool & Clinic directors & $x$ & & \\
\hline \multicolumn{5}{|l|}{ Exploratory } \\
\hline HIV treatment self-efficacy & Patients & $x$ & $x$ & $x$ \\
\hline Depression & Patients & $x$ & $x$ & $x$ \\
\hline HIV treatment retention & Patients & $x$ & $x$ & $x$ \\
\hline Substance use risk behaviors & Patients & $x$ & $x$ & $x$ \\
\hline
\end{tabular}

The Information-Motivation Behavioral Model [16] has informed the battery of measures. The following basic demographic data will be collected: age, gender, race, ethnicity, current living situation, marital status, sexual orientation, educational attainment, employment status, date of HIV diagnosis and ART initiation, mode of transmission, substance use diagnosis, and the intensity and duration of the substance use disorder. Primary outcomes will be feasibility and acceptability of the LS-DU and assessment procedures to be used in a larger trial. We will assess preliminary effectiveness of the LS-DU in a real-world clinical setting delivered by trained community-based clinicians. Effectiveness outcomes will include ART adherence measured by MEMScaps, viral load, and substance use measured by the 30-day Timeline Followback. Implementation outcomes will include readiness to adopt and qualitative data on barriers and facilitators to uptake. We will also gather exploratory data on the following potential moderators of the LS-DU intervention: HIV treatment self-efficacy, depression, HIV treatment retention, and HIV and substance use problem behaviors measured by the Addiction Severity Index (see Table 3 for measures).

We will evaluate the implementation context and sustainability potential of the LS-DU at the clinic level through surveys and semi-structured interviews. Individual interviews will be conducted post-intervention with clinicians and clinic leadership. Data will be collected regarding perceptions of providers and leadership concerning barriers/facilitators to intervention adoption, tools needed to deliver the intervention consistently, resources needed to maintain the intervention long-term, and adaptations needed to integrate into regular practice.
Providers and leadership will complete the Organizational Readiness for Implementing Change Scale [22] to examine organizational strengths/weaknesses that support sustainability of the intervention.

\section{Quantitative data analysis plan}

Considering this is a pilot project, analyses will have the goal of establishing feasibility and estimation of effect sizes, with modest expectations for rejection of the null hypotheses. As a first step, the equivalence of treatment condition assignment with regard to key baseline variables will be assessed on demographic characteristics, baseline ART adherence measures (self-report and viral load), drug category and addiction severity, and baseline levels of potential treatment mechanisms and moderators using tests of proportions or t-tests as appropriate. Should conditions differ on any characteristic; these variables will be used as covariates in outcome analyses. Other preliminary analyses will include patterns of missing data, research dropout rates, distributional properties of dependent and other measures, and correlations among outcome measures.

Hypotheses testing Given the developmental nature of this study, our primary goal is to establish feasibility and acceptability of the LS-DU and assessment procedures to be implemented in a future larger scale hybrid type 2 randomized trial. Our primary hypothesis is that participants completing and community clinicians delivering the LS-DU intervention will report high levels of acceptability of the intervention. Our secondary hypothesis is that the LS-DU intervention will demonstrate greater effectiveness than the health education outcome in terms 
of promoting days of adherence to HIV medication and reducing substance use. We will also collect exploratory data on implementation potential of the intervention and moderators of implementation effectiveness. Due to the small sample size, we will primarily be hoping to find a pattern of results that is supportive of the LS-DU rather than rigorously testing hypotheses to determine a stable effect size.

Primary outcomes: acceptability Participants' mean ratings of the program satisfaction questionnaire will be examined to determine level of satisfaction of the LS-DU.

Feasibility We will monitor the feasibility of the pilot trial by tracking recruitment, retention, and adherence rates of participants. We will assess feasibility of the intervention through qualitative interviews with the clinicians and a subset of patients.

Effectiveness We will measure treatment effectiveness via adherence to HIV medication (MEMScaps), viral load (eligibility screener vs. baseline vs. 6-months), and days of drug use (Timeline Followback). Separate Generalized Estimating Equation (GEE) models for adherence will be generated for proportion of days on which study medication was taken (as indicated by MEMScap) and the proportion of expected pills taken at the 1-, 3-, and 6-month follow-ups. Examining interactions between intervention and time will test differences in adherence over the course of the intervention across the intervention conditions. As our sample size limits our ability to conduct sophisticated longitudinal analyses, we will use GEE with caution and will revert to more standard general linear models should we encounter model convergence problems. Additional measures of treatment effectiveness, including selfreported adherence, viral load, and drug use, will also be analyzed using GEE.

Implementation context and sustainability potential Data will be collected at the clinic level through surveys and semi-structured interviews. Individual interviews will be conducted post-intervention with clinicians and clinic leadership. Data will be collected regarding perceptions of barriers and facilitators to intervention adoption, tools needed to deliver the intervention consistently, resources needed to maintain the intervention long-term, and adaptations needed to integrate into regular practice.

\section{Qualitative analysis}

Qualitative data will be analyzed using a thematic analysis framework and standard qualitative analysis techniques. An initial codebook will be developed from the interview guides and revised as themes emerge. Two coders will double-code all transcripts and discuss discrepancies until consensus is reached. A master codebook will be entered into NVivo 11. We will discuss the transcripts to analyze themes. A list of barriers and facilitators to intervention implementation and sustainability will be developed.

\section{Discussion}

HIV-infected PWUD continue to experience poorer rates of ART adherence, lapses in treatment retention, and increased morbidity and mortality [7, 8]. Existing adherence interventions have yet to prove effective among this high-risk population. This protocol seeks to improve ART adherence and promote linkage to substance use treatment by developing an adherence intervention tailored to HIV-infected PWUD. This behavioral intervention will utilize the syndemics framework to address critical factors aimed at improving adherence and reducing substance use. This study will use a pilot type 1 hybrid effectiveness-implementation design [19] given that Life Steps and BMI have both proven efficacious. In order to speed the translation of research to practice, we test the effectiveness of these interventions delivered in a realworld clinical setting, while simultaneously examining potential barriers and facilitators to implementation.

While this study will advance our understanding of treatment science and clinical practice with HIV-infected PWUD, there are a number of limitations which should be noted. First, since this is a pilot study the sample size is small and, therefore, will require testing in a larger trial to further examine effectiveness should this study produce a pattern of results that is favorable to the LS-DU intervention. Second, this is a brief, first-line intervention that aims to improve ART adherence and link HIV-infected PWUD to substance use treatment. Consequently, this will not be a comprehensive treatment intervention, and patients might require more intensive substance use intervention post-linkage to care. Third, participants will not be blind to study purpose and intervention condition due to the informed consent process and the nature of the content being addressed in each behavioral condition.

This research will result in the development of an ART adherence intervention tailored for PWUD and combined with a BMI aiming to link patients to appropriate substance use treatment services. Data from this study regarding implementation and sustainability potential will result in the development of an implementation manual for community-based HIV clinical settings. This work will inform a larger, multi-site study that places equal emphasis on both effectiveness and implementation outcomes. These results have the potential to make a significant impact on the management of ART adherence by improving the quality and utilization of 
evidence-based adherence interventions delivered to HIV-infected PWUD in the clinic setting. From both a clinical and a public health perspective, there is an urgent need to develop effective interventions to improve ART adherence and reduce substance use among HIVinfected people who use drugs. To our knowledge, no research has yet to adapt a brief, theory driven protocol that integrates HIV and substance use treatment among this high-risk population.

\section{Abbreviations}

HIV: human immunodeficiency virus; ART: antiretroviral therapy; PWUD: people who use drugs; PLWH: people living with HIV; CBT: cognitive behavior therapy; BMI: brief motivational interview; LS-DU: Life Steps-Drug Use.

\section{Authors' contributions}

KRC is the Principal Investigator for the study, led the conceptualization of the study and the drafting of the original grant proposal, and developed a first draft of the manuscript. SR, DO, SS, JR, and SB are Co-Mentors and contributed to conceptualization of the study. SB contributed to writing the initial draft of the manuscript. SR, DO, SS, JR, and SB also contributed to writing of the original grant proposal and successive drafts of the manuscript. All authors read and approved the final manuscript.

\section{Author details \\ 1 Department of Psychiatry, The University of Texas at Austin Dell Medical School, 1912 Speedway Austin, TX, USA. ${ }^{2}$ Department of Medicine, Alpert Medical School of Brown University, Providence, RI, USA. ${ }^{3}$ Department of Psy- chiatry and Human Behavior, Alpert Medical School of Brown University, Box G-BH, Providence, RI 02912, USA. ${ }^{4}$ Center for Alcohol and Addiction Studies, Brown University School of Public Health, 121 South Main Street, Box G-121-5, Providence, RI 02912, USA. ${ }^{5}$ Department of Psychology, University of Miami, Coral Gables, FL, USA. ${ }^{6}$ The Center for Prisoner Health and Human Rights, The Miriam Hospital, Providence, RI 02906, USA.}

\section{Acknowledgements}

Not applicable.

\section{Competing interests}

Dr. Safren receives royalties from Oxford University Press which publishes his intervention manual for Cognitive Behavioral Therapy for Adherence and Depression ("Coping with Chronic Illness") and includes the Life-Steps manual. The other authors declare that they have no competing interests.

\section{Availability of data and materials}

Not applicable.

\section{Consent for publication}

Not applicable.

\section{Ethics approval and consent to participate}

This study protocol has been approved by the Rhode Island Hospital Institutional Review Board (Protocol \#744017). Human subjects have been enrolled in phase 1 (qualitative interviews) of the study; however, formal data analysis has not been completed as of submission of this protocol.

\section{Funding}

The current protocol was funded by a Grant from NIDA (K23DA039037) awarded to the first author. Dr Rich's involvement has been supported by NIDA (K24DA022112) and NIAID (P30 Al42853). Dr. Safren was supported by NIDA (K24DA040489). The content is solely the responsibility of the authors and does not necessarily represent the official views of NIDA or the National Institutes of Health.

\section{Publisher's Note}

Springer Nature remains neutral with regard to jurisdictional claims in published maps and institutional affiliations.

Received: 15 August 2017 Accepted: 2 March 2018

Published online: 02 April 2018

\section{References}

1. Singer M, Clair S. Syndemics and public health: reconceptualizing disease in bio-social context. Med Anthropol Q. 2003;17(4):423-41.

2. Administration SAaMHS. Results from the 2010 National Survey on Drug Use and Health: Summary of National Findings, NSDUH series $\mathrm{H}-41, \mathrm{HHS}$ Publication No. (SMA) 11-4658. In: Services USDoHaH, edited. Rockville, MD; 2011.

3. Mimiaga MJ, Reisner SL, Grasso C, Crane HM, Safren SA, Kitahata MM, Schumacher JE, Mathews WC, Mayer KH. Substance use among HIVinfected patients engaged in primary care in the United States: findings from the centers for AIDS research network of integrated clinical systems cohort. Am J Public Health. 2013:103(8):1457-67.

4. Malta M, Strathdee SA, Magnanini MMF, Bastos Fl. Adherence to antiretroviral therapy for human immunodeficiency virus/acquired immune deficiency syndrome among drug users: a systematic review. Addiction. 2008;103(8):1242-57.

5. Lucas GM, Cheever LW, Chaisson RE, Moore RD. Detrimental effects of continued illicit drug use on the treatment of HIV-1 infection. J Acquir Immune Defic Syndr (1999). 2001;27(3):251-9.

6. Cunningham CO, Sohler NL, Cooperman NA, Berg KM, Litwin AH, Arnsten $\mathrm{JH}$. Strategies to improve access to and utilization of health care services and adherence to antiretroviral therapy among HIV-infected drug users. Subst Use Misuse. 2011:46(2-3):218-32.

7. Hinkin CH, Barclay TR, Castellon SA, Levine AJ, Durvasula RS, Marion SD, Myers HF, Longshore D. Drug use and medication adherence among HIV-1 infected individuals. AIDS Behav. 2007;11(2):185-94.

8. Knowlton AR, Arnsten JH, Eldred LJ, Wilkinson JD, Shade SB, Bohnert AS, Yang C, Wissow LS, Purcell DW. Antiretroviral use among active injectiondrug users: the role of patient-provider engagement and structural factors. AIDS Patient Care STDs. 2010;24(7):421-8.

9. Thompson MA, Aberg JA, Hoy JF, Telenti A, Benson C, Cahn P, Eron JJ Jr, Günthard HF, Hammer SM, Reiss P, et al. Antiretroviral treatment of adult HIV infection: 2012 recommendations of the International Antiviral Society-USA Panel. JAMA, J Am Med Assoc. 2012;308(4):387-402.

10. Sorensen JL, Haug NA, Larios S, Gruber VA, Tulsky J, Powelson E, Logan DP, Shapiro B. Directly administered antiretroviral therapy: pilot study of a structural intervention in methadone maintenance. J Subst Abuse Treat. 2012;43(4):418-23.

11. Kanters S, Park JJH, Chan K, Socias ME, Ford N, Forrest Jl, Thorlund K, Nachega JB, Mills EJ. Interventions to improve adherence to antiretroviral therapy: a systematic review and network meta-analysis. The Lancet HIV. 2017:4(1):e31-40.

12. Mathes T, Pieper D, Antoine SL, Eikermann M. Adherence-enhancing interventions for highly active antiretroviral therapy in HIV-infected patients—a systematic review. HIV Med. 2013;14(10):583-95.

13. Safren $S A$, O'Cleirigh CM, Bullis JR, Otto MW, Stein MD, Pollack MH. Cognitive behavioral therapy for adherence and depression (CBT-AD) in HIV-infected injection drug users: a randomized controlled trial. J Consult Clin Psychol. 2012;80(3):404-15.

14. Alfonso V, Toulson A, Bermbach N, Erskine Y, Montaner J. Psychosocial issues influencing treatment adherence in patients on multidrug rescue therapy: perspectives from patients and their health care providers. AIDS Patient Care STDs. 2009;23(2):119-26.

15. Jeste DV, Palmer BW, Appelbaum PS, Golshan S, Glorioso D, Dunn LB, Kim K, Meeks T, Kraemer HC. A new brief instrument for assessing decisional capacity for clinical research. Arch Gen Psychiatry. 2007;64(8):966-74.

16. Fisher JD, Fisher WA, Amico KR, Harman JJ. An information-motivationbehavioral skills model of adherence to antiretroviral therapy. Health Psychol. 2006;25(4):462-73. 
17. Moyers TB, Martin T, Manuel JK, Hendrickson SML, Miller WR. Assessing competence in the use of motivational interviewing. J Subst Abuse Treat. 2005;28(1):19-26.

18. Carroll KM, Nich C, Sifry RL, Nuro KF, Frankforter TL, Ball SA, Fenton L, Rounsaville BJ. A general system for evaluating therapist adherence and competence in psychotherapy research in the addictions. Drug Alcohol Depend. 2000;57(3):225-38.

19. Curran GM, Bauer M, Mittman B, Pyne JM, Stetler C. Effectiveness-implementation hybrid designs: combining elements of clinical effectiveness and implementation research to enhance public health impact. Med Care. 2012;50(3):217-26.

20. Stout RL, Wirtz PW, Carbonari JP, Del Boca FK. Ensuring balanced distribution of prognostic factors in treatment outcome research. J Stud Alcohol 1994;12:70-75
21. Fleming M, Brown R, Brown D. The efficacy of a brief alcohol intervention combined with \%CDT feedback in patients being treated for type 2 diabetes and/or hypertension. J Stud Alcohol. 2004;65(5):631-7.

22. Shea CM, Jacobs SR, Esserman DA, Bruce K, Weiner BJ. Organizational readiness for implementing change: a psychometric assessment of a new measure. Implement Sci IS. 2014;9:7-7.

23. Miller WR, Sanchez VC. Motivating young adults for treatment and lifestyle change. In: Howard GS, Nathan PE, editors. Alcohol use and misuse by young adults. Notre Dame, IN: University of Notre Dame Press; 1994. p. 51-81.

\section{Submit your next manuscript to BioMed Central and we will help you at every step:}

- We accept pre-submission inquiries

- Our selector tool helps you to find the most relevant journal

- We provide round the clock customer support

- Convenient online submission

- Thorough peer review

- Inclusion in PubMed and all major indexing services

- Maximum visibility for your research

Submit your manuscript at www.biomedcentral.com/submit 